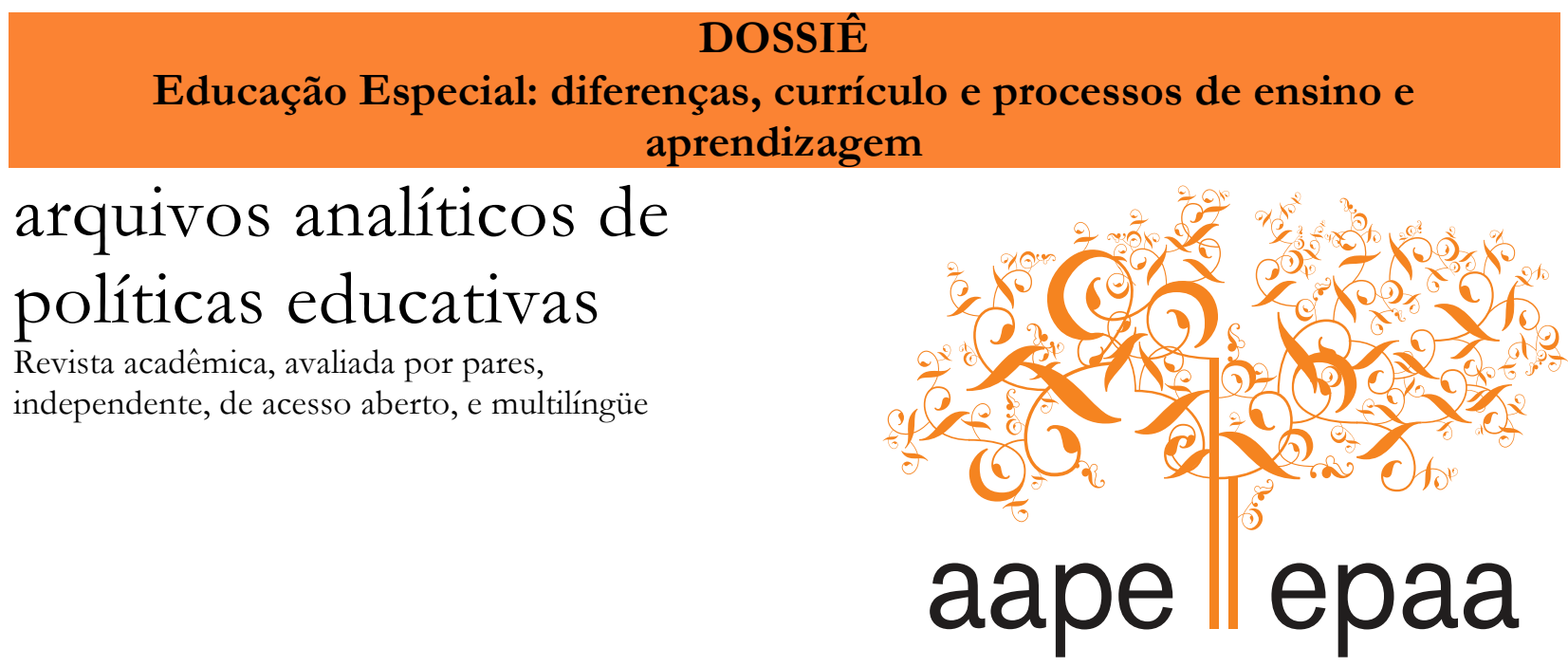

Arizona State University

Volume 22 Número $83 \quad 11$ de agosto de $2014 \quad$ ISSN 1068-2341

\title{
A Comunicação Alternativa para além das Tecnologias Assistivas
}

\author{
Leila Regina d'Oliveira de Paula Nunes \\ \& \\ Cátia Crivelenti de Figueiredo Walter \\ Universidade do Estado do Rio de Janeiro \\ Brasil
}

Citação: Nunes, L. R. O. P. \& Walter, C. C. F. (2014). A Comunicação Alternativa para além das Tecnologias Assistivas. Arquivos Analíticos de Políticas Educativas, 22(83). Dossiê Educação Especial: diferenças, currículo e processos de ensino e aprendizagem. Editoras convidadas: Márcia Denise Pletsch \& Geovana Mendonça Lunardi Mendes. http://dx.doi.org/10.14507/epaa.v22n83.2014

Resumo: A presença de alunos com paralisia cerebral e com autismo é crescente no cotidiano das escolas regulares. Professores frequentemente necessitam de formação no emprego de recursos de comunicação alternativa junto a esse alunado que exibe graves dificuldades de comunicação oral. Serão descritos estudos do Grupo de Pesquisa Linguagem e Comunicação Alternativa do Programa de Pós-Graduação em Educação de uma universidade publica conduzidos em escolas regulares e especiais. Serão apresentados e comentados recursos de Comunicação Alternativa elaborados com o propósito de favorecer as habilidades linguísticas, comunicativas e sociais desses alunos em suas interações em sala de aula. Finalmente, serão discutidas questões relativas à pesquisa em sala de aula, à concepção de comunicação e ao papel da tecnologia na Comunicação Alternativa.

Palavras-chave: Educação Especial; tecnologia assistiva; comunicação alternativa; formação inicial. 


\begin{abstract}
Alternative Communication beyond Assistive Technologies
Abstract: The number of students with cerebral palsy and autism enrolled in traditional schools is growing each day. Teachers frequently need to be trained to employ Alternative Communication resources with these students, who present severe difficulties in oral communication. The studies conducted in traditional and special schools by the Language and Alternative Communication Research Group from the Graduate Program in Education of a public university will be briefly described. Alternative Communication resources will be presented. A description of how these resources facilitate the students' linguistic, communicative, and social abilities will follow. Finally, issues related to the development of research in schools, the conception of communication, and the role of technology in Alternative Communication will be discussed.
\end{abstract}

Keywords: Special Education; assistive technology; alternative and augmentative communication; initial training.

\title{
La Comunicación Alternativa más allá de las Tecnologías de Asistencia
}

Resumen: La presencia de alumnos con parálisis cerebral y autismo está creciendo cada día en las escuelas regulares. Los maestros a menudo necesitan entrenamiento en el uso de recursos alternativos de comunicación para los alumnos que presentan serias dificultades en la comunicación oral. Se describirán brevemente los estudios realizados en escuelas regulares y especiales por el Grupo de Investigación: Lenguaje y Comunicación Alternativa del Programa de Postgrado de Educación de una universidad pública. Se presentarán y se comentarán las características de medios alternativos de comunicación, diseñados con el propósito de promover las habilidades lingüísticas, comunicativas y sociales de estos estudiantes en sus interacciones durante la clase. Por último, serán discutidas las cuestiones relacionadas con la investigación realizada en las escuelas, con la concepción de la comunicación y con el papel de la tecnología de asistencia en la Comunicación Alternativa

Palabras-clave: Educación Especial; tecnología de asistencia; comunicación alternativa; formación inicial

\section{A inclusão de alunos com deficiência nas escolas}

Na década de 1990, a Declaração Mundial sobre Educação para Todos, redigida na Tailândia, e a Declaração de Salamanca ressaltaram a urgência no atendimento aos direitos dos alunos com necessidades educacionais especiais. Em que pesem o amparo legal e os esforços dos pais e dos agentes educacionais para garantir o acesso, o ingresso e a permanência dessa população na escola básica, é essencial que se garanta igualmente o sucesso na aprendizagem dos conteúdos acadêmicos e não apenas a socialização (Mendes, 2006). Muitos elementos tem sido apontados como favorecedores da inclusão de alunos com deficiência. Dentre eles estão a formação continuada e qualificada de professores, o ensino e a consultoria colaborativos, o emprego consistente dos planos educacionais individualizados e a introdução da Tecnologia Assistiva (TA). A TA é uma área do conhecimento que se propõe a promover e ampliar habilidades em pessoas com limitações funcionais decorrentes de deficiência e do envelhecimento. Recursos que favorecem a comunicação, a adequação postural e a mobilidade, o acesso independente ao computador, a escrita alternativa, $o$ acesso diferenciado ao texto, são apenas alguns exemplos das inúmeras modalidades e possibilidades da TA (Schirmer, 2012). Com efeito, o acesso efetivo a serviços e recursos da TA e mais especificamente da Comunicação Alternativa são fundamentais no processo ensino-aprendizagem de alunos com deficiência, notadamente, aqueles com dificuldades acentuadas de comunicação que, de forma crescente, estão presentes nos bancos escolares (Schirmer, 2012; Nunes, Walter \& Schirmer, 2013). 
A Comunicação Alternativa (CA) é uma das áreas da Tecnologia Assistiva (TA) que atende pessoas sem fala ou escrita funcional ou em defasagem entre sua necessidade comunicativa e sua habilidade em falar e/ou escrever. Mais especificamente, Comunicação Alternativaenvolve o emprego de gestos manuais, posturas corporais, expressões faciais, uso de miniaturas, de símbolos gráficos (como fotografias, gravuras, desenhos e linguagem alfabética), de voz digitalizada ou sintetizada por meio dos quais pessoas sem fala articulada, devido a fatores psicológicos, neurológicos, emocionais, físicos e/ou cognitivos, podem efetuar a comunicação face a face (Nunes, 2003; Soto\& von Tetzchner, 2003).

\section{Formação de recursos humanos para introdução da Comunicação Alternativa nas escolas}

O Grupo de Pesquisa Linguagem e Comunicação Alternativa do Programa de Pós-Graduação em Educação de uma universidade pública vem se dedicando sistematicamente desde 1995 ao ensino e à pesquisa sobre a linguagem e a comunicação de pessoas com deficiência, em especial, aquelas com paralisia cerebral, autismo, deficiência intelectual severa, deficiência múltipla e surdocegueira. Foram desenvolvidos 14 projetos de pesquisa financiados pelo CNPq, FAPERJ, UERJ e CAPES, agrupados em oito eixos temáticos. Dentre eles se destacam aqueles voltados para investigar os programas de formação de recursos humanos para introduzir a comunicação alternativa nas escolas do ensino fundamental e de educação de jovens e adultos. Descreveremos a seguir esses estudos.

\section{Introdução da Comunicação Alternativa nas escolas}

Os principais objetivos de dois projetos de pesquisa financiados pelo CNPq (Nunes, 2009 a) e pela FAPERJ (Nunes, 2009, b) foram, por meio de um programa de formação de professores, introduzir e avaliar os efeitos da inclusão dos recursos de Comunicação Alternativa em duas salas de aula de uma escola especial do município do Rio de Janeiro e analisar o processo comunicativo de alunos com deficiência severa de comunicação oral com seus interlocutores na escola e em casa. A introdução dos recursos de comunicação alternativa foi conduzido através de uma variedade de atividades, todas elas planejadas durante as reuniões semanais da equipe de pesquisa com as professoras, a saber: a) leitura de textos sobre comunicação alternativa (CA); b) oferta de software Boardmaker para confecção de pictogramas; c) oferta de caixas de comunicação contendo pictogramas para os alunos; d) oferta de sugestões de pranchas de comunicação e de softwares para comunicação escrita; e) demonstração da utilização das pranchas para estabelecer conversação com os alunos. No entanto, o elemento-chave desse conjunto de procedimentos foi a observação e análise de trechos de sessões filmadas das professoras desenvolvendo atividades pedagógicas e interagindo com os alunos, conduzidas em sessões semanais de discussão com a professora e toda a equipe de pesquisa. Nessas ocasiões, as professoras eram convidadas a expressar suas opiniões o sobre seu próprio desempenho e o desempenho dos alunos e a ouvir os comentários dos demais participantes e principalmente trazer suas dúvidas e questões para o grupo. Esse procedimento de auto confrontação, descrito por pareceu se constituir em oportunidade de desenvolvimento, como uma tomada de consciência no sentido em que Vygotsky diz que ela é uma generalização: perceber as coisas de outra maneira consiste em adquirir outras possibilidades de ação em relação a elas. Generalizando um processo próprio de minha atividade, eu adquiro a possibilidade de uma outra relação com ela (Vygotsky, 1989). Após a problematização, a equipe formada pelos pesquisadores, mestrandos e doutorandos, com formação nas áreas de Fonoaudiologia, Terapia Ocupacional, Psicologia e Pedagogia traziam sugestões para a resolução dos problemas apontados, e planejavam a execução da solução que poderia ser realizada pelas professoras de sala de aula ou por um ou mais membros da equipe de apoio através de uma visita a sala de aula.Com o financiamento recebido das agencias de pesquisa, uma sala de aula da escola especial foi equipada com computador, monitor, 
teclado com colmeia, acionadores e softwares como o Intellytools, e o Boardmaker with Speaking Dinamically Pro destinados à impressão de imagens, adaptação de atividades pedagógicas e programação de pranchas dinâmicas. O software "Escrevendo com Simbolos" foi igualmente empregado para facilitar a elaboração de textos com imagens e adaptações de atividades de leitura. Com a impressora jato de tinta e a plastificadora, também disponibilizadas pelo projeto, as professoras imprimiam as atividades e pranchas de comunicação na própria sala de aula e plastificavam os pictogramas usados pelos alunos.

A figura 1 ilustra a professora Carmen ${ }^{1}$ utilizando pranchas de CA e recebendo orientação dos profissionais envolvidos no projeto.

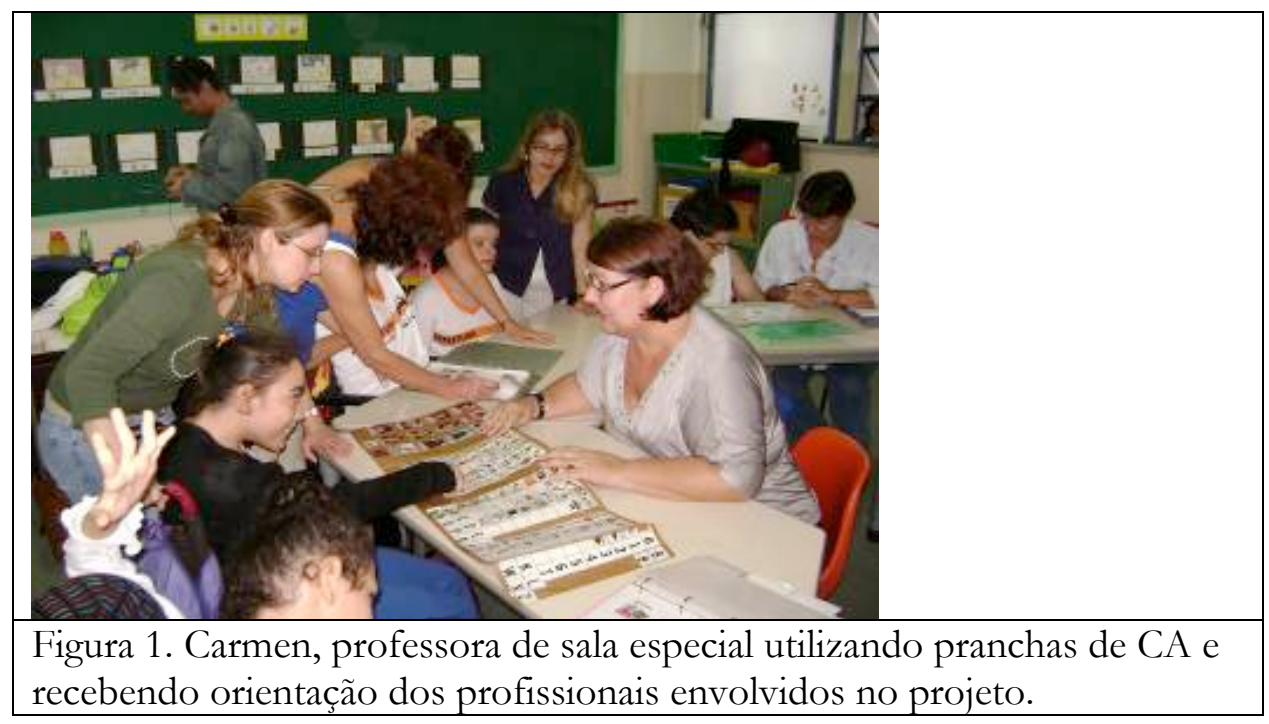

A equipe de pesquisa foi bem sucedida em ensinar às professoras a elaborar e a empregar tais recursos de baixa e alta tecnologia em uma diversidade de situações. Os dados coletados apontaram para uma mudança razoável nas habilidades comunicativas e sociais dos alunos em sala de aula e maior sensibilidade das professoras às questões de comunicação nesse contexto. A disposição dos alunos em sala de aula, sentados em semicírculo, a disponibilização de pranchas e cadernos de comunicação, o incentivo para a conversa dos alunos com os colegas e a maior agilidade na troca de turnos da professora com cada aluno favoreceu, de algum modo, maior participação nas interações e na expressão de pensamentos e sentimentos. Contudo, a despeito de tais efeitos, os alunos sem fala articulada desempenharam papel mais passivo nessas interações, como pode ser verificado pelo maior número de respostas às iniciativas de outros do que de interações iniciadas por eles próprios. Além disso, estiveram ausentes funções comunicativas mais nobres como a narrativa, o planejamento e o julgamento.

Ainda que gestos e pranchas com pictogramas tenham sido usados pelos alunos oralizados, pelas próprias professoras e pelas assistentes de pesquisa, a assimetria de papéis nas interações persistiu, ou seja, os interlocutores falantes assumiram frequentemente o domínio na situação, reforçando assim o papel passivo dos alunos não falantes. Com efeito, os dados da presente investigação confirmam os achados de outros estudos que mostram que é o adulto que verbaliza quem inicia e controla a interação estabelecendo os tópicos da conversação (nem sempre do interesse de seu parceiro), antecipando os desejos e completando as enunciados de seu interlocutor

1 Todos os nomes aqui mencionados são fictícios, com a finalidade de preservação da identidade dos participantes das pesquisas. 
(Light, Collier\& Parnes, 1985).

Muitas investigações têm mostrado que tipicamente as interações de pessoas que falam com aquelas que usam sistemas de comunicação alternativa tendem a seguir um padrão de pergunta e resposta. Os falantes solicitam informações através de perguntas fechadas ou mesmo de perguntas abertas especificas (Light etal., 1985) e os não falantes respondem frequentemente com mensagens telegráficas com restrito teor de informação (Lund \& Light, 2007). São igualmente observadas a alta frequência de turnos produzidas pelas numerosas questões de esclarecimento por parte dos interlocutores falantes (Nunes, Tubagi, Camelo, Magalhães, Almeida \& Paula, 2003). Tais conclusões se alinham com um estudo longitudinal conduzido por Light e colaboradores, no qual foram acompanhados sete indivíduos com paralisia cerebral que usavam a Comunicação Alternativa desde os 4-6 anos até completarem 19-23 anos. Os dados revelaram que embora capazes de solicitar informação a seus interlocutores, os sujeitos raramente o faziam. Tanto na infância quanto na idade adulta, a maioria dos turnos interacionais desses sujeitos consistia em responder de forma afirmativa ou negativa as perguntas formuladas por seus interlocutores ou em prover informações pontuais (Lund\& Light, 2007; Light, et al., 1985; Nunes, Brito, Santanna, Togashi, Walter \& Schirmer, 2010)

De toda forma, as professoras passaram a perceber que na escola, torna-se essencial o planejamento das atividades e dos materiais pedagógicos adaptados e das pranchas de comunicação. Para implementar com sucesso programas instrucionais junto a alunos especiais sem fala articulada, o professor precisa ter clareza do plano pedagógico e preparar seu plano de aula elaborando previamente todos os materiais e recursos necessários para um bom ensino a cada dia. É preciso lembrar que "a escola tem muitos papeis, mas o principal deles é ensinar. A escola não pode se tornar, apenas, espaço de convivência social ou espaço para fazer amizades. A escola tem de ensinar a todos e não apenas acolhê-los" (Pelosi, 2006, p. 131).

A formação dos participantes do projeto Acessibilidade comunicativa para alunos com deficiência: formação inicial de professores (Nunes, 2008), desenvolvido por Schirmer (2012), como sua tese de doutorado, foi fundamentada na Metodologia Problematizadora (Berbel, 1998; 1999). Os objetivos gerais da formação foram: iniciar a preparação dos graduandos da disciplina Pesquisa e Prática Pedagógica, oferecida em quatro semestres seguidos, para interagir com alunos sem fala articulada e/ou fala funcional; desenvolver habilidades de coletar dados, estruturar um estudo de caso e elaborar um plano de intervenção especializada. A formação objetivou, também, estimular parcerias entre o aluno da graduação, o aluno com deficiência, a família, os profissionais da escola e da Saúde na construção do planejamento e na resolução dos problemas. Os dados mostraram que houve modificações positivas nas atitudes e nas condutas dos graduandos ao interagir com os alunos especiais.

A figura 2a, à esquerda, mostra os alunos de graduação elaborando e utilizando recursos de Comunicação Alternativa e material pedagógico adaptado junto a uma criança com paralisia cerebral. A figura $2 \mathrm{~b}$, à direita, uma criança com autismo utilizando uma prancha de "sim" e "não" no Laboratório de Tecnologia Assistiva/Comunicação Alternativa (LATECA) do Programa de Pós Graduação em Educação de uma universidade pública. 


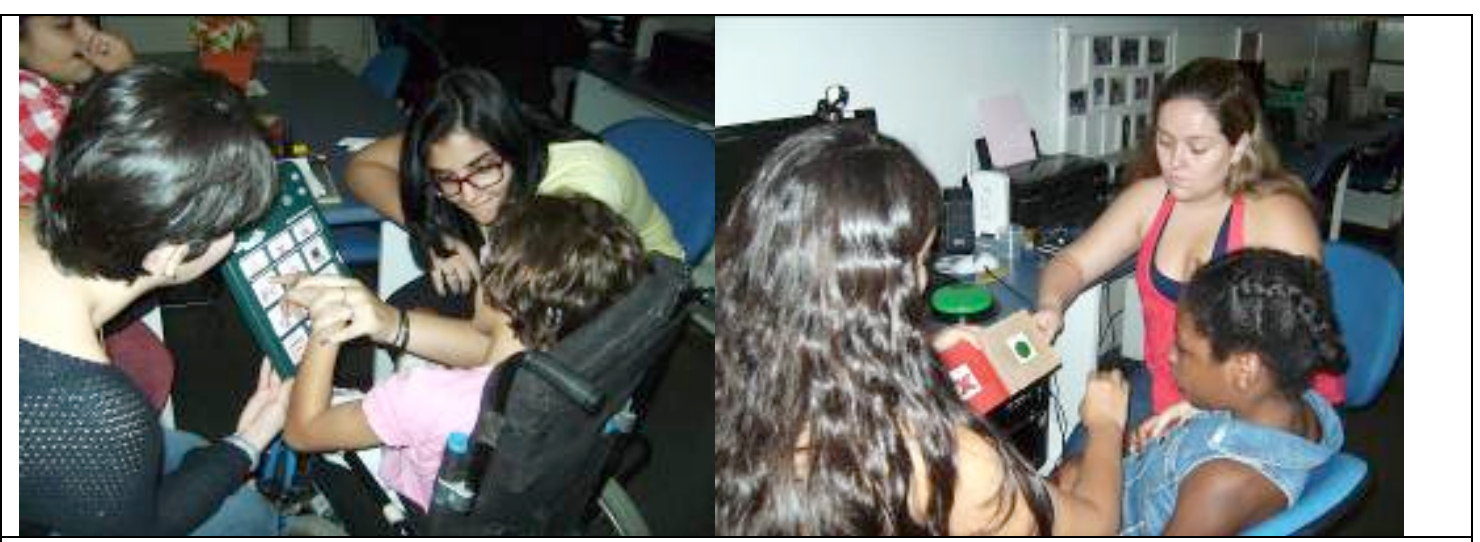

Figura 2a e 2b. Graduandos de Pedagogia e alunos especiais empregando diferentes pranchas de comunicação alternativa no LATECA

$\mathrm{Na}$ figura 3, as graduandas Aline e Jane conversam com a aluna Isabel, uma criança com paralisia cerebral, por meio do uso de uma prancha de Comunicação Alternativa.

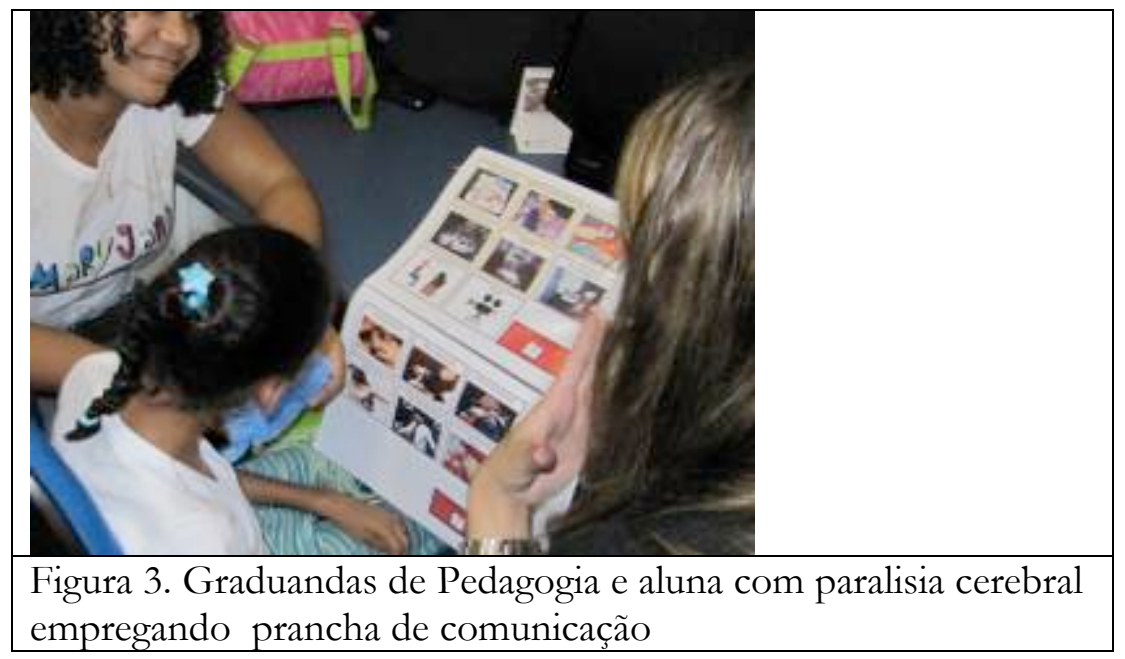

Um aspecto importante da formação dos graduandos era que, no início do trabalho, eles apresentavam dificuldade em identificar as respostas dos alunos especiais. Com frequência, os graduandos solicitavam que os alunos se expressassem, fazendo perguntas fechadas ou abertas, sem, contudo, esperar a resposta. Às vezes até respondiam por eles. Com o andamento do trabalho, os graduandos passaram a oferecer aos alunos mais oportunidades de manifestar opinião e fazer escolhas, apresentando modelos e pistas, fazendo varredura das opções de respostas gráficas e aguardando a expressão dos alunos especiais. Estes passaram a interagir com mais frequência com os graduandos, buscando-os para fazer solicitações, responder a perguntas e mediar relações com outro colega, professor ou familiar. Observou-se, igualmente, o crescimento no vocabulário contido nas pranchas de comunicação.

Os procedimentos de formação dos graduandos de Pedagogia dessa investigação têm sido empregados junto às novas turmas dessa disciplina. Um estudo em preparação revelou que esses alunos têm se beneficiado sobremaneira em seu desempenho como professores de alunos com severas deficiências na comunicação, exibindo competência e entusiasmo. 
O Projeto Tecnologia Assistiva para a inclusão comunicativa de alunos com deficiência: formação continuada de professores (Nunes, 2012)visou ensinar professores regulares e estagiários-mediadores de três escolas regulares e um centro especializado a planejar, implementar e avaliar atividades pedagógicas e materiais didáticos adaptados para atender alunos do ensino fundamental que apresentassem severos comprometimentos em sua comunicação oral, como aqueles com paralisia cerebral, autismo e deficiência múltipla. Araújo (2012) e Netto (2012) redigiram suas dissertações de mestrado sob nossa orientação abordando este tema.

$\mathrm{Na}$ figura 4, a professora da sala de recursos multifuncionais desenvolve junto à Duda, aluna com paralisia cerebral, uma atividade destinada a sistematizar códigos para respostas utilizando cartões de sim e não. Dessa forma, a aluna poderia responder às perguntas fechadas elaboradas pela professora da sala de aula regular durante atividades acadêmicas.

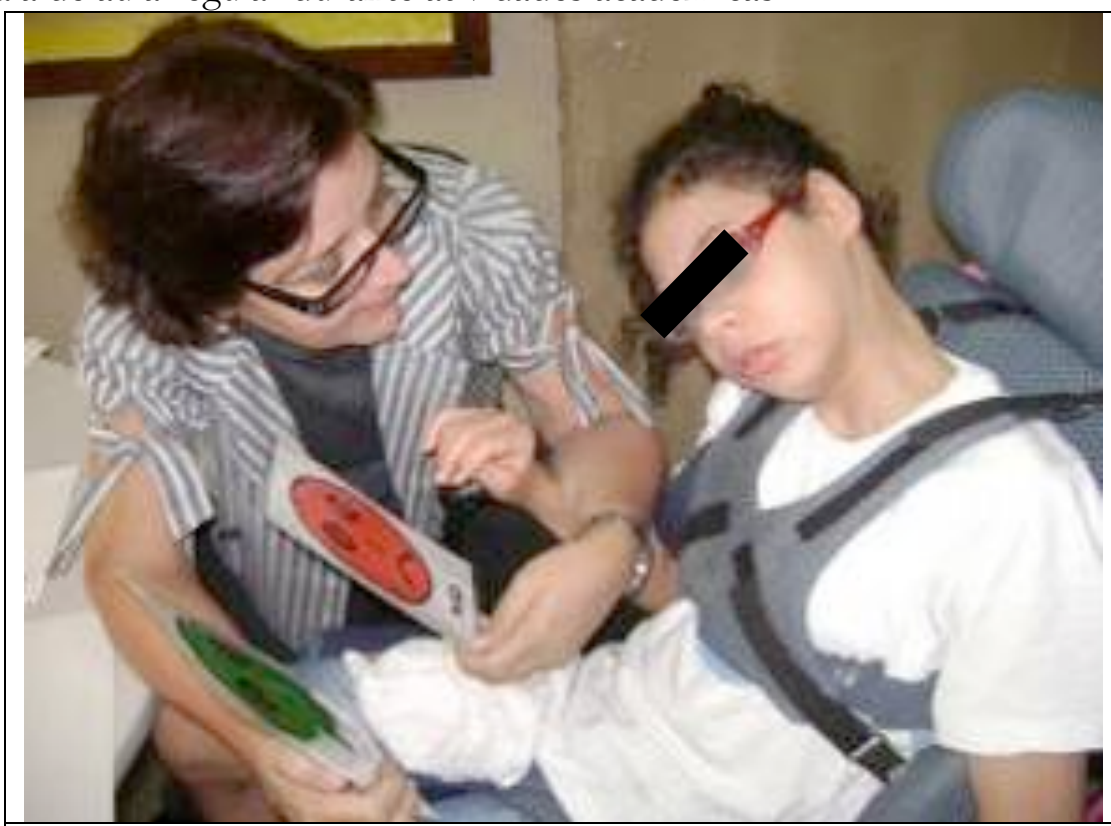

Figura 4. Duda, aluna incluída na sala regular, e a professora Cláudia utilizando cartões de comunicação alternativa.

A Figura 5 mostra uma adaptação pedagógica formalizada, realizada durante uma experiência de bidocência (dois professores de ensino especializado). Uma das professoras dinamiza a atividade de leitura e interpretação do livro de história "Vida Marinha", enquanto a outra usa cartões pictográficos como apoio na realização da atividade com a aluna Maria. 


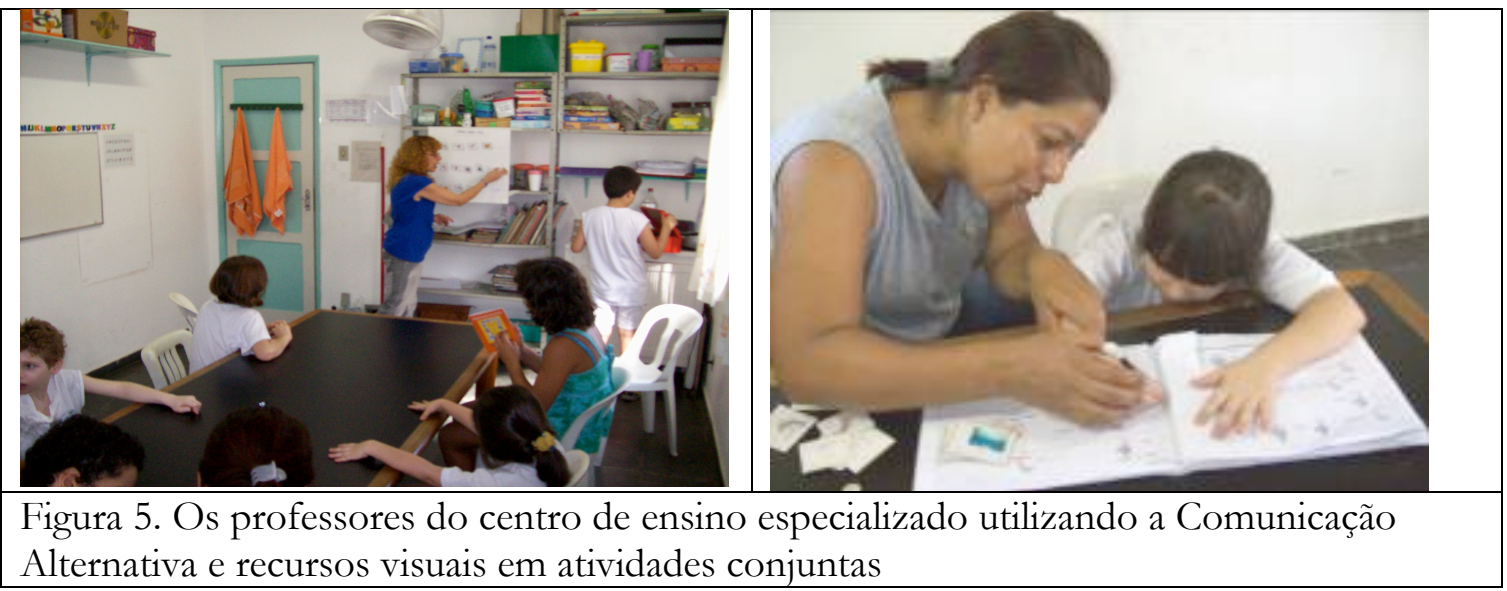

O projeto intitulado Quero conversar com você: Comunicação Alternativa para alunos com autismo no contexto escolar (Walter, 2013) teve como objetivo promover a aplicação e a avaliação de um programa de comunicação alternativa, baseado no sistema PECS-Adaptado (Walter, 2000) ${ }^{2}$, proposto para alunos com autismo incluídos no ensino regular no município do Rio de Janeiro. O projeto foi realizado em duas etapas, sendo a primeira destinada a capacitar 35 professores da rede regular de ensino do município do Rio de Janeiro que atuam em salas de recursos ou salas de Atendimento Educacional Especializado (AEE). Após a capacitação dos professores, foi possível verificar, pelos relatos dos mesmos, que havia necessidade de se utilizar o PECS-Adaptado com os alunos que não apresentam fala funcional e que o professor do AEE deveria ficar responsável pela coordenação e aplicação do programa nas escolas regulares. Na segunda etapa do projeto foi possível aplicar o programa de comunicação alternativa em quatro alunos com autismo, sendo dois em sala de aula regular e outros dois em salas de AEE.

As imagens, a seguir, revelam que os alunos aprenderam a se comunicar pelo intercâmbio de figuras, solicitando itens de interesse para as professoras durante atividades preparadas em sala de aula e na sala de recurso.

\footnotetext{
2 PECS-Adaptado é um sistema de comunicação alternativa que promove a comunicação alternativa por intercâmbio de figuras destinado a pessoas com autismo. Este sistema consiste numa adaptação do PECS [Picture Exchange Communication System - Bondy e Frost, 1994] ao contexto brasileiro e possui flexibilidade para os diferentes contextos: escolar, familiar e especializado.
} 


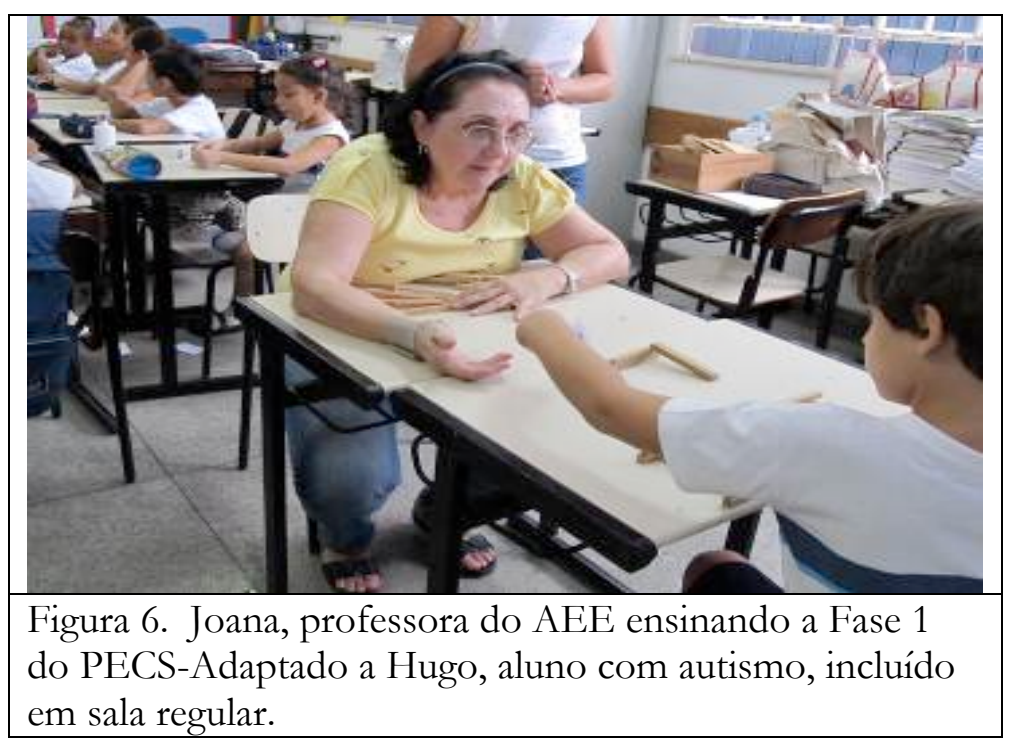

$\mathrm{Na}$ figura 6, a professora Joana, com a mão estendida, aguarda a entrega da figura com a imagem de "jogo" pelo aluno Hugo, expressando seu desejo de obter uma peça do seu jogo favorito. Esta etapa do programa de comunicação alternativa corresponde à Fase 1 do PECS-Adaptado e estava sendo realizada em sala de aula regular, onde futuramente, o aluno deverá solicitar seus itens desejados à professora regente da turma e aos colegas.

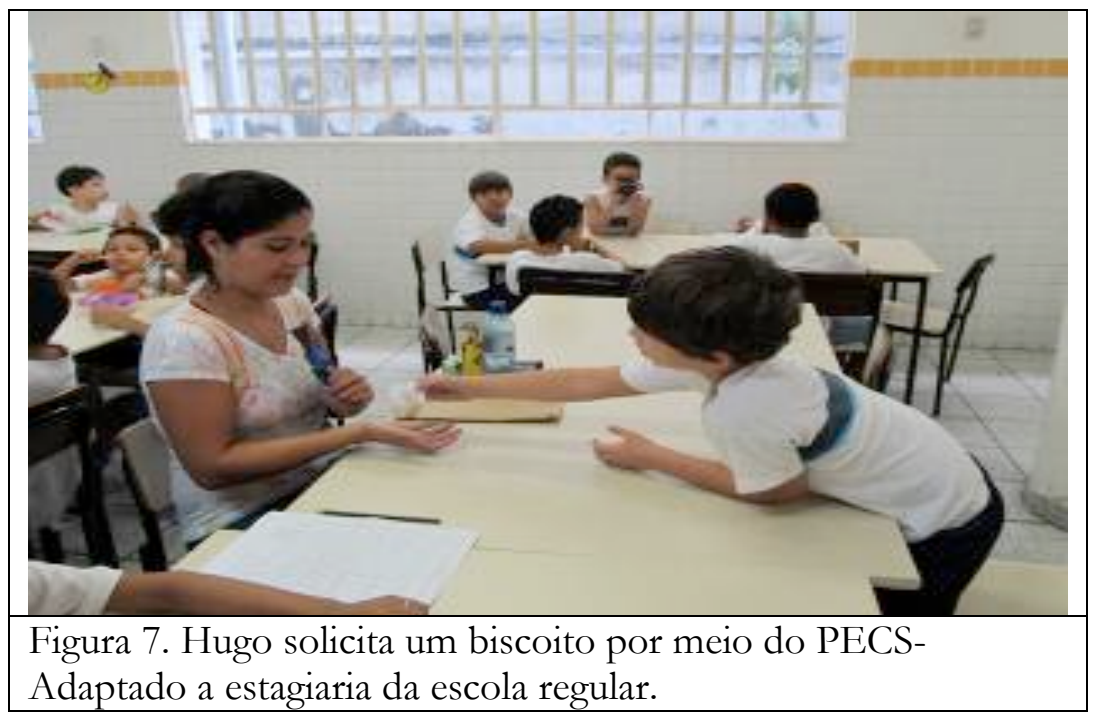

$\mathrm{Na}$ figura 7, o aluno Hugo solicita um biscoito à Tânia, uma estagiária do ensino regular, no refeitório junto a outros alunos, quando a professora do AEE não estava presente. Este evento atesta a generalização do uso dos cartões por Hugo em novos contextos e com outros interlocutores.

O programa de Comunicação Alternativa também foi aplicado pelas professoras de AEE em salas de recursos multifuncionais, onde o aluno foi ensinado a pedir seus itens de interesse à professora durante as atividades específicas, como revela a figura 8. 


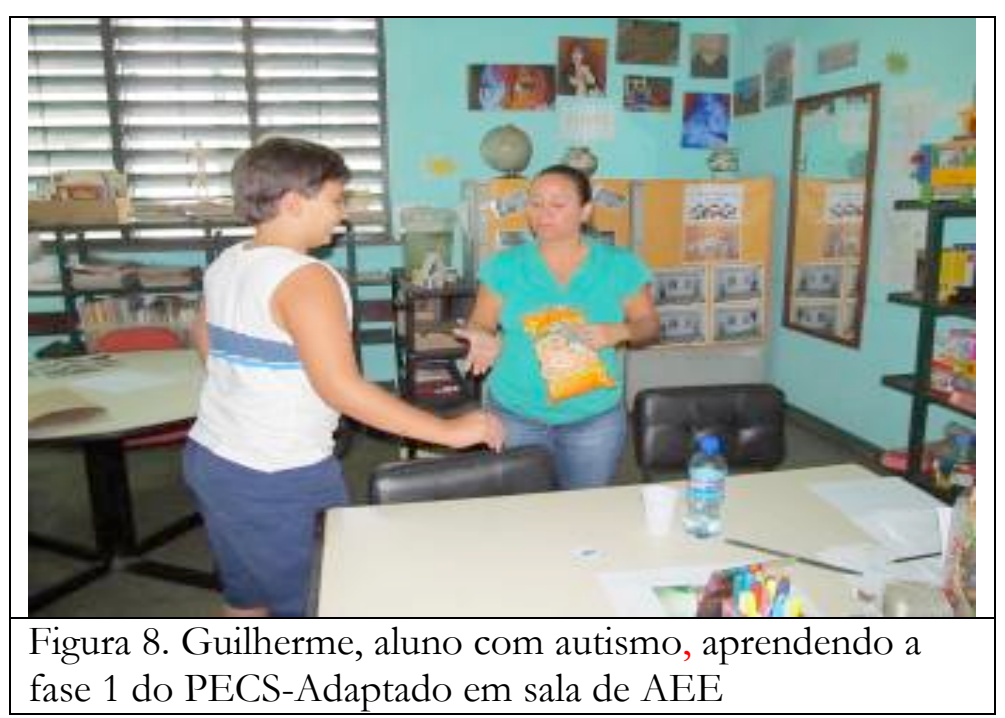

O uso dos recursos de comunicação alternativa no contexto regular de ensino possibilitou, em diversas situações e contextos, condições de comunicação mais eficaz e maior clareza na compreensão dos alunos, por meio da troca de figuras pelos itens desejados (Walter, Nunes \& Togashi, 2011)

\section{Discussão}

O desenvolvimento dos projetos acima descritos conduziu a reflexões em dois aspectos essenciais - a pesquisa na sala de aula e o papel da tecnologia assistiva na Comunicação Alternativa.

Conduzir pesquisas em escolas tem se constituído em grande desafio para o professor/pesquisador universitário. Nos estudos acima descritos, os esforços para introduzir inovações tecnológicas como os recursos de Comunicação Alternativa, de baixa, média ou de alta tecnologia, sempre envolveram confrontos entre pesquisadores e docentes de sala de aula. Costuma-se dizer, de forma apressada, que tais embates refletem a velha dicotomia teoria versus prática. Zeichner (1998), contudo, nos ensina que tal fato reflete o dissenso entre as teorias implícitas do professor e as teorias mais ou menos explicitas do pesquisador. É, com efeito, um embate entre ideias, concepções. As teorias cristalizadas implicitamente constituem entraves para práticas inovadoras no campo educacional. Uma grande dificuldade é a couraça com a qual se revestem professores e pesquisadores. O professor é aquele que tem que dominar o conhecimento do processo, do aluno, sendo desnecessário aprender algo mais. A receptividade à ideia de mudança precisa fazer parte das crenças daqueles que se envolvem em uma investigação. Zeichner (1998) alerta para preconceitos muito difundidos de que o corpo docente das escolas é pouco receptivo e não quer pensar e agir segundo novas alternativas; e de que alguns professores, como também, alguns acadêmicos, não estão interessados no desafio de mudar seus modos usuais de agir no mundo. Ao abordar essa separação entre teoria e prática docente, é preciso, sob uma perspectiva dialética, compreender que as teorias têm a função precípua de tensionar continuamente a prática, e é nela que as construções teóricas são checadas (Gamboa, 2003).Assim, é preciso considerar que conduzir pesquisa numa sala de aula demanda a construção de uma cumplicidade entre pesquisadores e professores. Nesse sentido, considera-se que o planejamento de momentos de troca, de estudo e de reflexão conjuntos sejam essenciais para que as partes envolvidas se percebam como sujeitos de uma pesquisa colaborativa de fato (Nunes, Walter \& Schirmer, 2013).

Nos dias atuais, as tecnologias estão presentes em quase todos os aspectos da vida cotidiana, inclusive na escola (Monteiro da Cruz \& Monteiro, 2013). As inovações tecnológicas têm se difundido de forma exponencial no campo da Comunicação Alternativa, com o desenvolvimento de softwares 
sofisticados, mouses adaptados, recursos de acionamento, pranchas com recursos de voz digitalizada, e mais recentemente com os tablets e I Pads e outras tecnologias móveis para a Comunicação Alternativa. O risco, contudo, com o encantamento com esses gadgets e consequente foco apenas na tecnologia é negligenciar o que realmente importa - a comunicação e a interação do usuário desses recursos e seus interlocutores (Light \& McNaughton, 2013).

A introdução da Comunicação Alternativa em sala de aula ou em qualquer outro ambiente da pessoa sem fala articulada leva-nos a refletir sobre em que consiste de fato a comunicação humana. Se concebermos a comunicação como um processo contínuo no qual interlocutores mutuamente corregulam seus comportamentos, isto é, alteram suas ações em função das ações atuais e antecipadas do parceiro, possibilitando a criação conjunta de sentido (Fogel, 1993), é essencial destacar a presença do outro. Em última análise, nos comunicamos para afetar o comportamento, o pensamento e o sentimento do nosso interlocutor e com ele negociarmos o sentido de nosso ato comunicativo. Assim, para que a pessoa desprovida de fala se comunique, não basta a disponibilização de recursos de Comunicação Alternativa, como pranchas e cadernos de comunicação, comunicadores portáteis, computadores e softwares especiais. Nem todos os aspectos da comunicação podem ser substituídos quando os recursos da Comunicação Alternativa são utilizados. A ênfase na interação humana deve anteceder qualquer procedimento ou técnica, pois comunicar é uma ação existencial e vital na relação humana. Na emissão da mensagem está implícita a relação com o interlocutor, ressaltando assim que a comunicação não é feita somente de conteúdos, mas também de aspectos emocionais, de movimentos e expressões faciais que complementam e direcionam o rumo da conversa em questão. Assim, tão ou mais importante do que o emprego dos recursos tecnológicos, sejam eles de alto ou baixo custo, é a presença do interlocutor interessado e preparado para acolher e responder às mensagens da pessoa não falante e que respeite e utilize, ele próprio, essas modalidades alternativas de comunicação.

O processo de aquisição de competência comunicativa não depende somente da inserção de linguagens alternativas na rotina das pessoas, mas implica mudança de atitude dos interlocutores frente à diversidade de possibilidades para tornar a comunicação entre duas pessoas significativa e efetiva. Isto significa que, não é apenas inserir um código comunicativo, mas favorecer a ampliação e encorajar necessidades emergentes de comunicação e o uso de funções comunicativas mais sofisticadas. É preciso lembrar, em suma, que competência comunicativa não é um traço intrapessoal, mas um constructo interpessoal (Lund \&Light,1997; Soro-Camats, 2003; von Tetzchner \& Martinsen, 2000).

\section{Referências}

Araújo, C. A G. (2012). O ensino colaborativo favorecendo a inclusão de alunos com deficiência física e transtorno global do desenvolvimento nas escolas municipais do Rio de Janeiro. Dissertação de mestrado defendida no Programa de Pós-Graduação em Educação, Universidade do Estado do Rio de Janeiro. Rio de Janeiro.

Berbel, N. A. N. (1998). A problematização e a aprendizagem baseada em problemas. Interface Comun Saúde Educ., v.2, p. 139-154. http:/ / dx.doi.org/10.1590/S1414-32831998000100008 , (1999) A metodologia da problematização e os ensinamentos de Paulo Freire: uma relação mais que perfeita. In: N. A. N Berbel (Org) Metodologia da problematização: fundamentos e aplicações (pp.1-28). Londrina: EDUEL.

Bondy, A. \& Frost, L. (1994). PECS: The Picture Exchange Communication System. Cherry Hill, NJ: Pyramid Educational Consultants, Inc. 
Clot, Y. ; Faïta, D. ; Fernandez, G. \& Scheller,. L. (2000) . Entrevistas em auto confrontação cruzada: um método em clínica da atividade. In http://pettnt/v2n1/articles/v2n1a3.htm

Fogel, A. (1993). Developing through relationships: Origins of communication, self, and culture. Chicago: University of Chicago Press.

Gamboa, S. (2003). A contribuição da pesquisa na formação docente. In: A. M. Reali \&M. G. Mizukami (Orgs.), Formação de professores: tendências atuais (PP. 115-130) São Carlos: EdufsCar.

Light, J.\& McNaughton, D. (2013). Putting people first: Re-thinking the role of technology in Augmentative and Alternative Communication intervention. Augmentative and Alternative Communication, 29 (4), 299-309. http://dx.doi.org/10.3109/07434618.2013.848935

Light, J.; Collier, B. \& Parnes, P. (1985). Communicative interaction between young non-speaking physically disabled children and their primary caregivers: Part II - Commmunicative functions. Augmentative and Alternative Communication, 1 (1), 98-106. http:/ /dx.doi.org/10.1080/07434618512331273591

Lund, S.\&Light, J. (2007). Long - term outcomes for individuals who use augmentative and alternative communication: Part II - Communication interaction. Augmentative and Alternative Communication, 23 (1), 1- 15. http:/ /dx.doi.org/10.1080/07434610600720442

Monteiro da Cruz, M. \& Monteiro, A. (2013). Acessibilidade cognitiva para o letramento de jovens com deficiência intelectual. Arquivos Analiticos de Políticas Educativas, 21(74). Recuperado [data] http:/ / epaa.asu.edu/ojs/article/view/1326 Dossiê Educação de Jovens e Adultos.

Netto, M. M. F. C. (2012). A Comunicação Alternativa e a aprendizagem de crianças com autismo, Asperger e Angelman: formação continuada de profissionais de Educação e Saúde. Dissertação de mestrado defendida no Programa de Pós-Graduação em Educação, Universidade do Estado do Rio de Janeiro. Rio de Janeiro.

Nunes, L. R. P (2012) Tecnologia assistiva para a inclusão comunicativa de alunos com deficiência: formação continuada de professores. Relatório de projeto de pesquisa aprovado pela FAPERJ (proc. 26/110047/2010).

(2009 a) Dando a voz através de imagens: comunicação alternativa para indivíduos com deficiência. Relatório de projeto de pesquisa aprovado pela FAPERJ (proc. 26/110235/2007). (2009 b) Promovendo a inclusão comunicativa de alunos não oralizados com paralisia cerebral e deficiência múltiplaRelatório de projeto de pesquisa aprovado pelo CNPq (proc. 473360/2007-1). (2003). Linguagem e Comunicação Alternativa: Uma introdução. In: Nunes, L. R. Favorecendo o desenvolvimento da comunicação em crianças e jovens com necessidades educacionais especiais. (pp. 1-13). Rio de Janeiro: Dunya.

Nunes, L. R. P. ; Brito, D. A.; Santanna, S. R. S. ; Togashi, C. M. ; Walter, C. C. F. \& Schirmer, C. (2010). Como alunos sem fala articulada se comunicam em sala: estudo descritivo das modalidades e funções comunicativas. In: E. G. Mendes; M. A. Almeida. (Org.). Das margens ao centro: perspectivas para as políticas e práticas educacionais no contexto da educação especial inclusiva. (Vol 1 - 191-202) 1ed.Araraquara: Junqueira \& Marin.

Nunes, L. R. P.; Walter, C. C. F. \& Schirmer, C. R. (2013). Comunicação Alternativa: passaporte para a inclusão escolar. In M. Leite, C., Guerreiro, A. M., Medeiros, R. (Orgs) Tecnologias Assistivas - Experiências e Desafios (pp. 17-24).Mossoró, RN: Editora da Universidade Estadual do Rio Grande do Norte.

Pelosi, M. (2006). Por uma escola que ensine e não apenas acolha recursos e estratégias para a inclusão escolar. In E. Manzini (Org) Inclusão e acessibilidade (pp. 121-132). Marília: Associação Brasileira de Pesquisadores em Educação Especial.

Schirmer, C. (2012). Metodologia Problematizadora na formação de graduandos de Pedagogia em Comunicação Alternativa. Tese de Doutorado defendida no Programa de Pós-Graduação em Educação da Universidade do Estado do Rio de Janeiro. 
Soro-Camats, E. (2003). Uso de ajudas técnicas para a comunicação, o jogo, a mobilidade e o controle do meio: uma abordagem habilitadora. In: C. B. Almirall, E. Soro-Camats, C.R. Bultó (Orgs.). Sistemas de sinais e ajudas técnicas para a comunicação alternativa e a escrita: princípios teóricos e aplicações (pp. 23-41) São Paulo: Livraria Santos Editora.

Soto, G. \& von Tetzchner, S. (2003). Supporting the development of alternative communication through culturally significant activies in shared educational settings. In: S. von Tetzchner e N. Grove (Orgs), Augmentative an alternative communication developmental issues (pp 287-299). London: Whurr.

von Tetzchner, S. \&Martinsen, H. (2000).Competências de conversação. In: S. von Tetzchner \& H. Martinsen (Eds.). Introdução à comunicação aumentativa e alternativa(pp.235-259.Porto: Porto Editora.

Vygotsky, L. (1989). Pensamento e linguagem. (J. L. Camargo, Trad.). S. Paulo: Martins Fontes.

Walter, C. C. F. (2000). Os efeitos da adaptação do PECS ao curriculum funcional natural em pessoas com autismo infantil. Dissertação de Mestrado. Programa de Pós-Graduação em Educação Especial, Universidade Federal de São Carlos, São Carlos.

(2013). Quero conversar com você: comunicação alternativa para alunos com autismo no contexto escolar. Relatório de projeto de pesquisa aprovado pela FAPERJ(proc. 110.106/2011). ; Nunes, L. R. ; Togashi, C. M . (2011) Quero conversar com você: comunicação alternativa para alunos com autismo no contexto escolar. In: Leila Regina Nunes; Miryam Pelosi; Catia Crivelenti Walter. (Org.). Compartilhando experiências: Ampliando a comunicação alternativa. 1ed. Marilia: Editora da Associação Brasileira de Pesquisadores em Educação Especial, 2011, v. 1, pp. 149-160.

Zeichner, K. (1998). Para além da divisão entre professor-pesquisador e pesquisador acadêmico. In: C. Geraldi, D. Fiorentini \& E.M. Pereira (Orgs.). Cartografias do trabalho docente (pp.207-236). Campinas: Mercado de Letras. 


\section{Sobre as Autoras}

\section{Leila Regina d'Oliveira de Paula Nunes}

Professora titular em Educação Especial do Programa de Pós Graduação em Educação e da Faculdade de Educação - Universidade do Estado do Rio de Janeiro leilareginanunes@terra.com.br

Possui Bacharelado em Psicologia e Formação de Psicólogo pela Universidade Federal do Rio de Janeiro (1970), mestrado em Special Education na George Peabody College, USA (1977) e doutorado (Ph D) em Special Education - Vanderbilt University (1985). Foi docente da Universidade Federal de S. Carlos e da Universidade Federal do Rio de Janeiro. É bolsista de produtividade em pesquisa do CNPq e Cientista do Nosso Estado pela FAPERJ. Tem experiência em Educação e Psicologia, atuando em áreas como desenvolvimento humano, linguagem e comunicação, interação social, comunicação alternativa, educação de pessoas com deficiência e metodologia experimental de pesquisa. Coordena o Laboratório de Tecnologia Assistiva e Comunicação Alternativa da Faculdade de Educação da UERJ. É sócia da International Society for Augmentative and Alternative Communication e sócia emérita da Associação Brasileira de Pesquisadores em Educação Especial. Coordenou mais de 15 projetos de pesquisa financiados pelo CNPq, CAPES, FAPESP e FAPERJ e orientou quase 50 teses e dissertações. É revisora de vários periódicos qualificados e autora de vários trabalhos científicos publicados em periódicos qualificados e em diversos livros. Foi organizadora dos seguintes livros: Pesquisa em Educação Especial na PósGraduação, Coletâneas da ANPEPP: Prevenção e Intervenção em Educação Especial, Favorecendo o desenvolvimento da comunicação em crianças e jovens com necessidades educacionais especiais, Um retrato da comunicação alternativa no Brasil: Relatos de pesquisas e experiências, Comunicar é Preciso: em busca das melhores práticas na educaşão do aluno com deficiência, Compartilhando experiências: Ampliando a comunicação alternativa, Ensaios sobre autismo e deficiência múltipla e Novas trilhas no modo de faz̧er pesquisa em Educação Especial.

\section{Cátia Crivelenti de Figueiredo Walter}

Professora Adjunta do Programa de Pós Graduação em Educação na linha de pesquisa em Educação Inclusiva e Processos Educacionais e do Departamento de Educação Inclusiva e Continuada da Faculdade de Educação - Universidade do Estado do Rio de Janeiro catiawalter@gmail.com

a autora é graduada em Fonoaudiologia, possui mestrado e doutorado em Educação Especial (UFSCar) e Pós-doutorado em Comunicação Alternativa (UERJ). Atua como pesquisadora e orientadora no grupo de pesquisa em Linguagem e Comunicação Alternativa do Programa de Pós Graduação em educação da UERJ. Tem experiência na área de fonoaudiologia clínica e Educação Especial, com ênfase em formação acadêmica, docência, principalmente nos seguintes temas: desenvolvimento e avaliação de linguagem, educação especial, autismo, pessoas com deficiência intelectual, paralisia cerebral, distúrbios de linguagem, técnicas de ensino especializado, PECS-Adaptado, comunicação alternativa e capacitação de familiares e de professores do ensino regular e especial. Coordena vários projetos de pesquisa com financiamento da Fundação de Amparo a pesquisa do Rio de Janeiro (FAPERJ). Organizou junto com a Profa. Leila Nunes e colegas os livros: Comunicar é Preciso: em busca das melhores práticas na educação do aluno com deficiência, Compartilhando experiências: Ampliando a comunicação alternativa, Ensaios sobre autismo e deficiência múltipla, além de ter publicado mais de 20 capítulos de livros e 6 artigos em periódicos conceituados na área da Educação Especial. 


\section{Sobre as Editoras Convidadas}

\section{Márcia Denise Pletsch}

Professora do Programa de Pós-Graduação em Educação, Contextos Contemporâneos e Demandas Populares (PPGEduc) na linha de pesquisa Estudos Contemporâneos e Práticas Educativas e do Departamento Educação e Sociedade da Universidade Federal Rural do Rio de janeiro. marciadenisepletsch@gmail.com

É pesquisadora na área de Educação Especial, atuando na formação de professores e de novos pesquisadores. É líder do Grupo de Pesquisa (CNPq) Observatório de Educação Especial e inclusão escolar: práticas curriculares e processos de ensino e aprendizagem e, por meio de convênio interinstitucional entre a UFRRJ e a Universidade do Estado do Rio de Janeiro (UERJ), também é líder do grupo de pesquisa Inclusão e aprendizagem de alunos com necessidades educacionais especiais: práticas pedagógicas, cultura escolar e aspectos psicossociais. Atualmente, coordena o Programa Observatório da Educação da CAPES com projeto de pesquisa em rede na área de deficiência intelectual envolvendo a Universidade Estadual de Santa Catarina (UDESC), Universidade do Vale do Itajaí (UNIVALI); e coordena também pesquisas financiadas pela FAPERJ na área de deficiência múltipla. É autora do livro "Repensando a inclusão escolar: diretrizes políticas, práticas curriculares e deficiência intelectual" e, em colaboração com Rosana Glat, do livro "Inclusão escolar de alunos com necessidades especiais". Organizou em parceria com outros pesquisadores, entre outros, os livros "Estratégias educacionais diferenciadas para alunos com necessidades especiais" e "Educação Especial e inclusão escolar: reflexões sobre o fazer pedagógico". Tem mais de vinte artigos publicados em revistas científicas nacionais e internacionais.

Geovana Mendonça Lunardi Mendes - Professora do Programa de Pós-Graduação em Educação e do Departamento de Pedagogia da Universidade do Estado de Santa Catarina (PGEUDESC). geolunardi@gmail.com

É pesquisadora na área de Educação Especial e dos Estudos Curriculares. Realizou Pós-Doutorado na Argentina e nos Estados Unidos da América, na área de Currículo e Novas Tecnologias, na Universidad de San Andres em Buenos Aires e em Ashland University, em Ohio. É pesquisadora coordenadora de diferentes projetos de investigação e participa como pesquisadora convidada em projetos de pesquisa nacionais e internacionais. Suas pesquisas e produções têm sido voltadas para área de Currículo e práticas escolares, em especial, as questões relativas as mudanças, novas tecnologias e inovações curriculares no espaço escolar, e também as práticas curriculares voltadas a inclusão de sujeitos com deficiência. Atualmente é a Coordenadora Nacional do Consórcio "Educação e Diversidade" do programa CAPES. FIPSE de Cooperação Internacional, envolvendo a Universidade Federal Rural do Rio de Janeiro, no Brasil e Georgetown College, Ashland University e Brighman Young University nos Estados Unidos e também do Projeto de Pesquisa: Aulas conectadas: mudanças curriculares e aprendizagem colaborativa nas escolas do PROUCA em Santa Catarina, com financiamento do CNPq e do Projeto Observatório de Práticas Escolares com financiamento da FAPESC. É coordenadora do Programa de Pós-graduação em Educação, Mestrado e Doutorado, da FAED, UDESC. Coordena o Observatório da Educação: Tablets, Computadores e Laptops, aprovado no Edital OBEDUC/CAPES. Entre suas atuais produções podem ser destacadas o livro intitulado "Objetos Pedagógicos: uma experiência inclusive em oficinas de Artes", em parceria com mais duas autoras e a organização do Livro "Deficiência e Escolarização: novas perspectivas de analise", hoje na segunda edição. É autora de inúmeros capítulos e artigos publicados em periódicos. 


DOSSIÊ
Educação Especial: diferenças, currículo e processos de ensino e
aprendizagem

\section{(c)}

SORERIGHISRESERVED O Copyright e retido pelo/a o autor/a (ou primeiro co-autor) que outorga o direito da primeira publicação à revista Arquivos Analíticos de Políticas Educativas. Más informação da licença de Creative Commons encontram-se em http://creativecommons.org/licenses/by-nc-nd/2.5. Qualquer outro uso deve ser aprovado em conjunto pelo/s autor/es e por AAPE/EPAA. AAPE/EPAA é publicada por Mary Lou Fulton Institute Teachers College da Arizona State University. Os textos publicados em AAPE são indexados por CIRC (Clasificación Integrada de Revistas Científicas, Espanha) DIALNET (Espanha),Directory of Open Access Journals, Education Full Text (H.W. Wilson), EBSCO Education Research Complete, , ERIC, , QUALIS A2 (Brasil), SCImago Journal Rank; SCOPUS, SOCOLAR (China). Contribua com comentários e sugestões a http://epaa.info/wordpress/ ou para Gustavo E. Fischman fischman@asu.edu.

Curta a nossa comunidade EPAA's Facebook https://www.facebook.com/EPAAAAPE e Twitter feed@epaa_aape. 


\section{arquivos analíticos de políticas educativas conselho editorial}

Editor: Gustavo E. Fischman (Arizona State University) Editores Associados: Rosa Maria Bueno Fisher e Luis A. Gandin

(Universidade Federal do Rio Grande do Sul)

Dalila Andrade de Oliveira Universidade Federal de Minas Gerais, Brasil

Paulo Carrano Universidade Federal Fluminense, Brasil

Alicia Maria Catalano de Bonamino Pontificia Universidade Católica-Rio, Brasil

Fabiana de Amorim Marcello Universidade Luterana do Brasil, Canoas, Brasil

Alexandre Fernandez Vaz Universidade Federal de Santa Catarina, Brasil

Gaudêncio Frigotto Universidade do Estado do Rio de Janeiro, Brasil

Alfredo M Gomes Universidade Federal de Pernambuco, Brasil

Petronilha Beatriz Gonçalves e Silva Universidade Federal de São Carlos, Brasil

Nadja Herman Pontificia Universidade Católica -Rio Grande do Sul, Brasil

José Machado Pais Instituto de Ciências Sociais da Universidade de Lisboa, Portugal

Wenceslao Machado de Oliveira Jr. Universidade Estadual de Campinas, Brasil
Jefferson Mainardes Universidade Estadual de Ponta Grossa, Brasil

Luciano Mendes de Faria Filho Universidade Federal de Minas Gerais, Brasil

Lia Raquel Moreira Oliveira Universidade do Minho, Portugal

Belmira Oliveira Bueno Universidade de São Paulo, Brasil

António Teodoro Universidade Lusófona, Portugal

Pia L. Wong California State University Sacramento, U.S.A

Sandra Regina Sales Universidade Federal Rural do Rio de Janeiro, Brasil

Elba Siqueira Sá Barreto Fundação Carlos Chagas, Brasil

Manuela Terrasêca Universidade do Porto, Portugal

Robert Verhine Universidade Federal da Bahia, Brasil

Antônio A. S. Zuin Universidade Federal de São Carlos, Brasil 


\section{education policy analysis archives editorial board}

Editor Gustavo E. Fischman (Arizona State University)

Associate Editors: Audrey Amrein-Beardsley (Arizona State University), Rick Mintrop, (University of California, Jeanne M. Powers (Arizona State University)

Jessica Allen University of Colorado, Boulder

Gary Anderson New York University

Michael W. Apple University of Wisconsin, Madison

Angela Arzubiaga Arizona State University

David C. Berliner Arizona State University

Robert Bickel Marshall University

Henry Braun Boston College

Eric Camburn University of Wisconsin, Madison

Wendy C. Chi* University of Colorado, Boulder

Casey Cobb University of Connecticut

Arnold Danzig Arizona State University

Antonia Darder University of Illinois, UrbanaChampaign

Linda Darling-Hammond Stanford University

Chad d'Entremont Strategies for Children

John Diamond Harvard University

Tara Donahue Learning Point Associates

Sherman Dorn University of South Florida

Christopher Joseph Frey Bowling Green State University

Melissa Lynn Freeman* Adams State College

Amy Garrett Dikkers University of Minnesota

Gene V Glass Arizona State University

Ronald Glass University of California, Santa Cruz

Harvey Goldstein Bristol University

Jacob P. K. Gross Indiana University

Eric M. Haas WestEd

Kimberly Joy Howard* University of Southern California

Aimee Howley Ohio University

Craig Howley Ohio University

Steve Klees University of Maryland

Jaekyung Lee SUNY Buffalo
Christopher Lubienski University of Illinois, UrbanaChampaign

Sarah Lubienski University of Illinois, UrbanaChampaign

Samuel R. Lucas University of California, Berkeley

Maria Martinez-Coslo University of Texas, Arlington

William Mathis University of Colorado, Boulder

Tristan McCowan Institute of Education, London

Heinrich Mintrop University of California, Berkeley

Michele S. Moses University of Colorado, Boulder

Julianne Moss University of Melbourne

Sharon Nichols University of Texas, San Antonio

Noga O'Connor University of Iowa

João Paraskveva University of Massachusetts, Dartmouth

Laurence Parker University of Illinois, UrbanaChampaign

Susan L. Robertson Bristol University

John Rogers University of California, Los Angeles

A. G. Rud Purdue University

Felicia C. Sanders The Pennsylvania State University

Janelle Scott University of California, Berkeley

Kimberly Scott Arizona State University

Dorothy Shipps Baruch College/CUNY

Maria Teresa Tatto Michigan State University

Larisa Warhol University of Connecticut

Cally Waite Social Science Research Council

John Weathers University of Colorado, Colorado Springs

Kevin Welner University of Colorado, Boulder

Ed Wiley University of Colorado, Boulder

Terrence G. Wiley Arizona State University

John Willinsky Stanford University

Kyo Yamashiro University of California, Los Angeles

* Members of the New Scholars Board 


\section{archivos analíticos de políticas educativas consejo editorial \\ Editor: Gustavo E. Fischman (Arizona State University)}

Editores. Asociados Alejandro Canales (UNAM) y Jesús Romero Morante (Universidad de Cantabria)

Armando Alcántara Santuario Instituto de Investigaciones sobre la Universidad y la Educación, UNAM México

Claudio Almonacid Universidad Metropolitana de Ciencias de la Educación, Chile

Pilar Arnaiz Sánchez Universidad de Murcia, España

Xavier Besalú Costa Universitat de Girona, España Jose Joaquin Brunner Universidad Diego Portales, Chile

Damián Canales Sánchez Instituto Nacional para la Evaluación de la Educación, México

María Caridad García Universidad Católica del Norte, Chile

Raimundo Cuesta Fernández IES Fray Luis de León, España

Marco Antonio Delgado Fuentes Universidad Iberoamericana, México

Inés Dussel FLACSO, Argentina

Rafael Feito Alonso Universidad Complutense de Madrid, España

Pedro Flores Crespo Universidad Iberoamericana, México

Verónica García Martínez Universidad Juárez Autónoma de Tabasco, México

Francisco F. García Pérez Universidad de Sevilla, España

Edna Luna Serrano Universidad Autónoma de Baja California, México

Alma Maldonado Departamento de Investigaciones Educativas, Centro de Investigación y de Estudios Avanzados, México

Alejandro Márquez Jiménez Instituto de Investigaciones sobre la Universidad y la Educación, UNAM México

José Felipe Martínez Fernández University of California Los Angeles, USA
Fanni Muñoz Pontificia Universidad Católica de Perú

Imanol Ordorika Instituto de Investigaciones Economicas - UNAM, México

Maria Cristina Parra Sandoval Universidad de Zulia, Venezuela

Miguel A. Pereyra Universidad de Granada, España

Monica Pini Universidad Nacional de San Martín, Argentina

Paula Razquin UNESCO, Francia

Ignacio Rivas Flores Universidad de Málaga, España

Daniel Schugurensky Arizona State University

Orlando Pulido Chaves Universidad Pedagógica Nacional, Colombia

José Gregorio Rodríguez Universidad Nacional de Colombia

Miriam Rodríguez Vargas Universidad Autónoma de Tamaulipas, México

Mario Rueda Beltrán Instituto de Investigaciones sobre la Universidad y la Educación, UNAM México

José Luis San Fabián Maroto Universidad de Oviedo, España

Yengny Marisol Silva Laya Universidad Iberoamericana, México

Aida Terrón Bañuelos Universidad de Oviedo, España

Jurjo Torres Santomé Universidad de la Coruña, España

Antoni Verger Planells University of Amsterdam, Holanda

Mario Yapu Universidad Para la Investigación Estratégica, Bolivia 